\title{
Relationships of the Dexamethasone Suppression Test to Clinical Severity and Degree of Melancholia
}

\author{
Anand Kumar. Kirsten Alcser, Leon Grunhaus, and John F. Greden
}

\begin{abstract}
Investigators continue to debate whether the Dexamethasone Suppression Test (DST) reffects clinical severity or degree of melancholia ("endogeneit."). To cvaluate this question, we studied 73 drug-free inpatients diagnosed with Schedule for Affective Disorders and SchizophrenialResearch Diagnostic Criteria (SADS/RDC) as having major depressive disorder (MDD). We compared absolute and dichotomous DSI values (DSI suppression versus nonsuppression) with absolute and dichotomous measures of endogeneity (as measured by operationally defined RDC items) and with Hamilton Rating Scale for Depression (HRSD) scores that were collected immediately prior to treatmen. We found that ( 1 ) degree of endogeneity correlated moderately $(\mathrm{r}=0.27)$ but significanth $(\mathrm{p}=0.02)$ with absolute DST values; (2) DST nonsuppression increased proportionateh with changes in categorical endogenous subtype $10 \%$ of the nonendogenous patients were nonsuppressives, $52 \%$ of probable endogenous. and $61 \%$ of subjects definitely endogcnous): (3) mean values for maximum DST concentrations increased steadily with catc. gorical endogeneity (nonendogenous, $1.44 \mu \mathrm{g} / \mathrm{dl}$; probable endogenous. $7.65 \mu \mathrm{g} / \mathrm{dl}$ : definite, $10.93 \mu \mathrm{gidl}: \mathrm{p}=0.0 \mathrm{l}):(4)$ HRSD scores correlated more strongly $(\mathrm{r}=0.45$ $\mathrm{p}=0.000)$ with maximum DST levels than did the degree of endogeneity. Age and weight changes did not account for the relationship of endogeneity to DST ralues. These data suggest that maximum postdexamethasone plasma cortisol levels reflect owerall severn of depression and endogeneity and that endogeneity per se is highl confounded with severits.
\end{abstract}

\section{Introduction}

The dexamethasone suppression test has been claimed to specifically reflect endogenous features of depression ("melancholia") (Carroll et al. 1981; Carroll 1982), but there in no consensus about this fact (Coryell et al. 1982). Despite uncertainty, few studies have assessed the relationship of absolute postdexamethasone cortisol levels to either the degree of endogeneity or severity of depressive features. Thus, the degree to which the DST correlates with either severity or endogeneity is still unknown.

From the Clinical Studies Unit for Atfective Disorders. Department of Psychiatry. University of Michugan Medical Conter Ann Arbor. Ml

Supported in part by NHH Grants MH28294 and MH39593 and by the University of Michigan, Mental Health Research Instutute and Department of Psychiatry.

Address reprint requests to Dr. John F. Greden. Clinical Studies Unit for Affective Disordera. Department af Psychatr. University of Michigan Medical Center. Ann Arbor. MI 48169

Received April 16. 1985. revised Octoher 16. 1985 
Various attempts have been made to delineate "specific clinical features" that occur with DST nonsuppression. Carroll et al. (1976) and Schlesser et al. (1979) failed to find any manifestations that convincingly distinguished nonsuppressors from suppressors. Winokur et al. (1978) and Lewis and Winokur (1983) reported that nonsuppression was more frequently associated with distinct genetic subtypes of depression, but others have challenged this finding (Carroll et al. 1980; Rudorfer et al. 1982). Reus (1982) suggested that nonsuppressors have more "classical endogenous signs of dysfunction" on admission, and more subjective complaints than suppressors. Nasr et al. (1983) also found that the DST discriminated between clinical subtypes.

A major problem in most prior reports is that associations between DST nonsuppression and endogeneity have been evaluated only in dichotomous subgroups, e.g., DST suppressors versus nonsuppressors or endogenous versus nonendogenous depressives. Unfortunately, considerable information is lost when categorical analyses constitute the sole approach; relationships between absolute hypothalamic-pituitary-adrenal (HPA) values and severity or degree of endogeneity may be missed.

To help clarify this somewhat controversial question, we examined the relationship between endogeneity and absolute postdexamethasone plasma cortisol levels. We hypothesized that as the absolute severity of the depressive syndrome and/or the number of endogenous symptoms increased, DST cortisol levels also would increase.

\section{Methods}

\section{Subjects}

Our sample consisted of 73 patients hospitalized in the Clinical Studies Unit (CSU) for Affective Disorders at the University of Michigan during 1979-1984. Each individual was evaluated with the standard CSU protocol: (1) a 10-14-day drug washout period, (2) comprehensive medical and neurological examination, (3) differential, absolute blood counts, thyroid studies, $B_{12}$ and folate levels, and VDRL, (4) electrocardiogram (EKG), electroencephalogram (EEG), chest and skull x-ray, (5) at least two independent, unstructured clinical evaluations by CSU psychiatrists, (6) a structured assessment by a trained interviewer using the Schedule for Affective Disorders and Schizophrenia (SADS) (Spitzer and Endicott 1975), (7) a family interview whenever feasible to confirm family history and longitudinal course, and (8) a review of prior medical records if available. At completion of these data collections, a consensus diagnosis was formulated using Research Diagnostic Criteria (RDC) (Spitzer et al. 1977). All patients were diagnosed as having major depressive disorder (MDD) using RDC. Clinical and demographic characteristics of the patient sample are shown in Table 1.

\section{Operational Definitions of Endogeneity}

Because a primary objective was to determine if DST values reflected degree of endogeneity, it was essential to have close temporal linkage between DSTs and measures of endogeneity. To accomplish this, we selected the DST from the time period immediately prior to treatment (10-14 days drug-free), thus controlling for sources of variance due to treatment. SADS interviews were not always conducted at the exact time when the drug-free DST was performed, but the Hamilton Rating Scale for Depression (HRSD) and the CSU current status forms were completed on the same day. Thus, to determine 


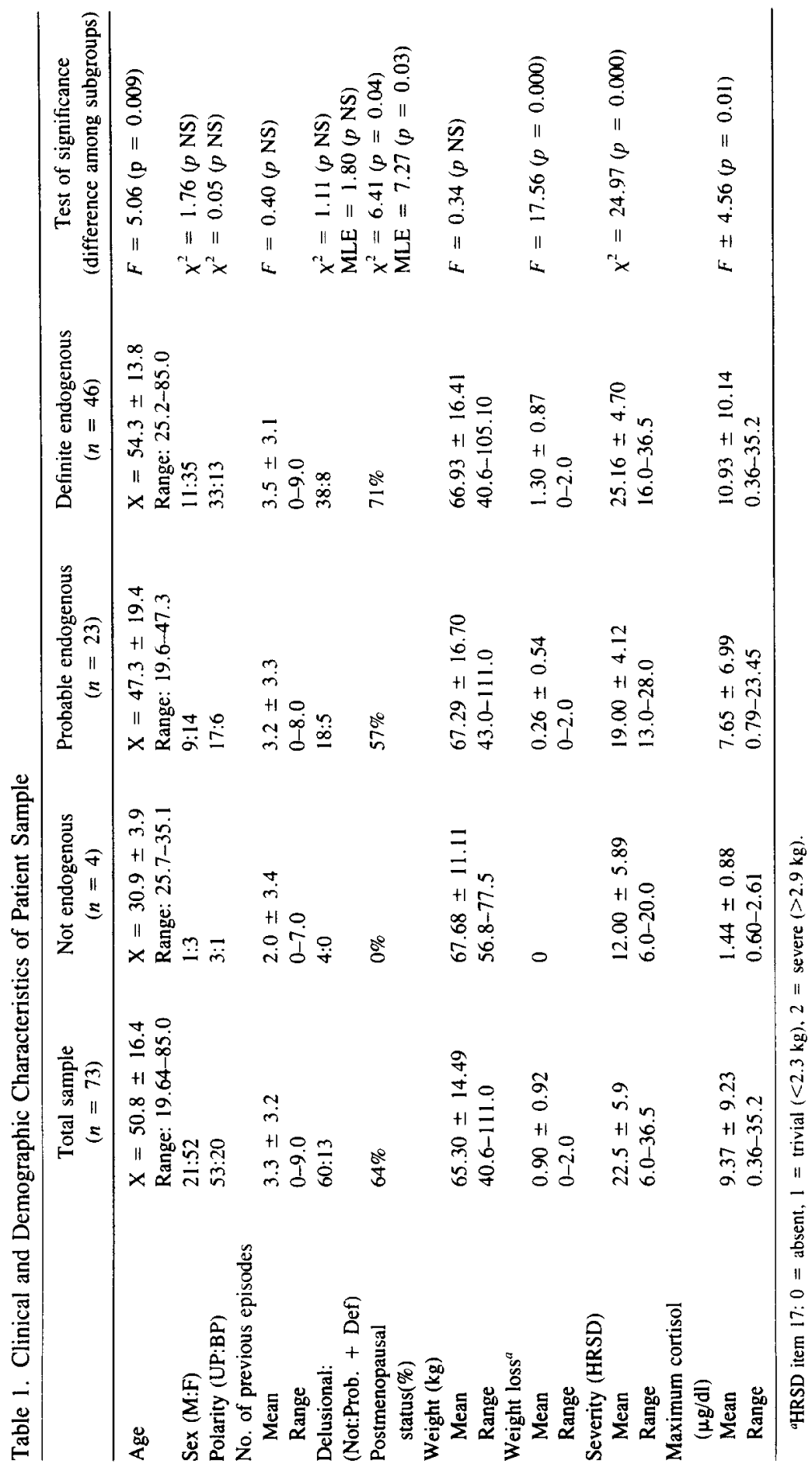


the degree of endogeneity, we developed an operational index of "endogeneity" to match the endogeneity items from the RDC. The comparisons are illustrated in Table 2 . The index was compiled using the Hamilton Rating Scale for Depression (Hamilton 1960) and the CSU Current Status form (this is a clinical questionnaire that clinicians on the unit complete on a weekly basis). Using this index, it was possible to compare postdexamethasone cortisol values and endogeneity at the same time point. We then divided subjects into three categories, based on the number of endogenous items they manifested, i.e., nonendogenous ( $0-3$ items), probable endogenous ( $4-6$ items), and definite endogenous (6 items and more). We thus developed measures of both categorical endogenous groups and actual degree of endogeneity (based on the number of endogenous symptoms). In compiling our "endogeneity" index and classifying patients into the three categories described above, we paid primary attention to the absolute number of endogenous symptoms. Groupings of symptoms (e.g., Group A versus Group B in RDC) were neither considered mandatory nor were they given any extra significance.

Clinical raters in the CSU always are blind to DST results.

\section{DST Technique}

We used our previously standardized approach for the DST (Carroll et al. 1981). All patients received $1 \mathrm{mg}$ of oral dexamethasone at 11:00 PM, and plasma samples were collected the following day at 4:00 PM and 11:00 PM. Plasma cortisol concentrations were assayed using a modification of Murphy's competitive protein binding technique (Murphy, 1967). Our intraassay coefficient of variance is $6.5 \%$ and interassay coefficient of variation is $9 \%$. In the CSU, a cortisol value $>5 \mu \mathrm{g} / \mathrm{dl}$ is considered to be "nonsuppression." This cut-off value was established in our setting only after comparisons of endogenous depressives, psychiatric controls, and normal controls. In our studies, the maximum cortisol level from either the 4:00 PM or 11:00 PM samples was used as the indicator of HPA dysregulation. This value should not be adopted indiscriminately in other settings.

Table 2 .

RDC-Endogenous signs and symptoms

Operational Index of Endogeneity (compiled from the Hamilton Rating Scale for depression and the Current Status questionnaire)

At least four symptoms are required for probable and six for definite, with at least one symptom from Group A.

Group A

(1) Distinct quality to depressed mood

(2) Lack of reactivity to environmental changes

(3) Mood is regularly worse in the morning

(1) Depressed mood

(2) Loss of reactivity Group $B$

(4) Feelings of self-reproach or excessive or inappropriate guilt

(5) Psychomotor retardation or agitation

(6) Poor appetite

(7) Weight loss

(8) Loss of interest or pleasure in usual activities or decreased sexual drive

(9) Early moming awakening or middle insomnia

(3) Diurnal mood change

(4) Guilt feelings

(5) Retardation and agitation

(6) Decreased sexual interest and loss of appetite

(7) Weight loss

(8) Loss of pleasure

(9) Middle and delayed insomnia 
The DST was repeated weekly. For this report, we used DST values immediately prior to treatment, following 10-14 days of drug washout. Maximum postdexamethasone plasma cortisol concentration (at 4:00 PM or 11:00 PM) and nonsuppression versus suppression were used as continuous and dichotomous DST variables, respectively.

\section{Data Analysis}

To improve normality of distribution and equality of variance, we log-transformed plasma dexamethasone concentrations prior to any statistical analyses. To compare subgroups on categorical dimensions, we used the $\left(\chi^{2}\right)$ test and the maximum likelihood estimate (MLE) (for tables larger than $2 \times 2$ with 0 cells). The one-way Analysis of Variance (ANOVA) was employed for analyses of differences among levels of endogeneity. A one-way Analysis of Covariance model (ANCOVA), with weight and age as covariates, also was used.

\section{Results}

There was a statistically significant relationship $(r=0.27 . p=0.02)$ between maximum postdexamethasone cortisol level and the degree of endogeneity (Figure 1). Consistent with this correlation, when the three categorical endogenous subgroups (non, probable. and definite) were examined, the percentage of nonsuppressors increased with endogeneity from $0 \%$ for the nonendogenous to $52 \%$ for the probable endogenous, to $61 \%$ for the definite endogenous (Table 3). The mean of maximum cortisol in each of the three subgroups also increased with endogeneity (Table 3). These observations establish a link between degree of endogeneity and postdexamethasone plasma levels. Thus, our hypothesis was supported. given the constraints placed on it by the small size ( $n=4$ ) of our nonendogenous group.

When we evaluated severity relationships with DST values, we found that DST values were even more strongly correlated with severity of depression (as demonstrated by HRSD scores) $(r-0.15, p=0.000)$ than they were with degree of endogeneity (see Figure 2 ).

Figure 1. The relationship between the number of endogenous sympoms (RDC) and the mean postdexamethasone plasma cortisol level $(\mu \mathrm{g} / \mathrm{dl})$. The relationship is a positive one. As the number of endogenous symptoms inereases, the postdexamethasone plasma cortisol level increases $(r=0.27$ $p=0.02$ ). In parentheses are the numbers of subjects in each group.

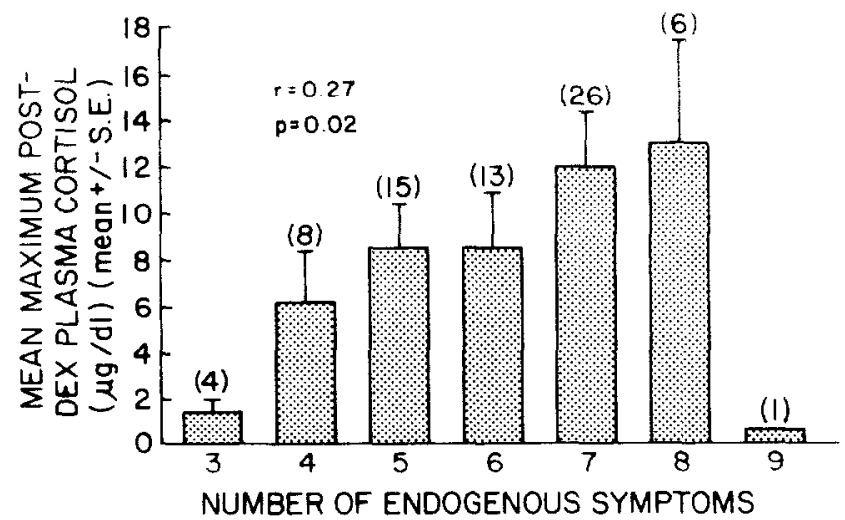


Table 3. Relationship of Categorical Endogenous Groups with Postdexamethasone Plasma Cortisol Level and Percent Nonsuppression

\begin{tabular}{|c|c|c|c|c|}
\hline \multirow{2}{*}{$\begin{array}{c}\text { Categorical } \\
\text { endogenous } \\
\text { groups }\end{array}$} & \multirow[b]{2}{*}{$n$} & \multicolumn{2}{|c|}{$\begin{array}{l}\text { Postdexamethasone plasma } \\
\text { cortisol }(\mu \mathrm{g} / \mathrm{d})^{a} \\
\end{array}$} & \multirow{2}{*}{$\begin{array}{c}\text { DST nonsuppression } \\
(\%)^{b}\end{array}$} \\
\hline & & Mean & SD & \\
\hline Not & 4 & 1.44 & \pm 0.88 & 0 \\
\hline Probable & 23 & 7.65 & \pm 6.99 & 52 \\
\hline Definite & 46 & 10.93 & \pm 10.14 & 61 \\
\hline
\end{tabular}

${ }^{a} F=4.56, p=0.01$.

${ }^{b} \mathrm{MLE}=7.11, p=0.03$.

We then evaluated the associations between "endogeneity" as described in the RDC and total HRSD items and found a significant correlation $(r=0.68, p=0.000)$. Thus, endogeneity per se is highly confounded with severity of depression. Indeed, the items on the HRSD that we did not consider to be conventionally "endogenous" also correlated significantly with postdexamethasone cortisol levels ( $r=0.49, p=0.000$ ).

The two endogenous and the nonendogenous subgroups did not differ significantly in terms of gender distribution, proportion of unipolar to bipolar, number of previous episodes, or presence of delusions (Table 1).

Age and weight loss have been reported to confound the relationship between DST and severity. One-way ANOVA revealed that patients who were definitely endogenous were older and reported greater weight loss (HRSD item 17) in this episode (Table 1). However, a one-way ANCOVA model, testing for the effect of group membership (endogeneity) on DST and treating age and weight loss as covariates, showed that this effect was not influenced by the covariates. When absolute weight was a covariate, however, we found that although a significant relationship existed between DST and this covariate $(F=11.96, p<0.001)$, group membership continued to have a significant effect in the prediction of DST $(F=4.49, p=0.01$ ). Thus, endogeneity remained a significant predictor of DST when absolute weight was held constant.

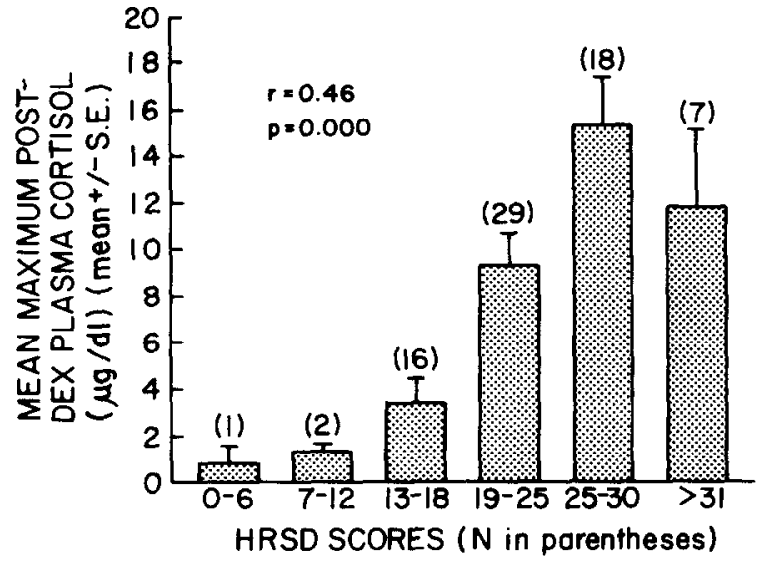

Figure 2. The relationship of the overall severity of depression as estimated by the Hamilton Rating Scale for Depression scores and the mean postdexamethasone plasma cortisol level $(\mu \mathrm{g} / \mathrm{dl})$. Subjects were divided into six categories based on their HRSD scores (each category signifying a range of the HRSD scores). The relationship of severity to postdexamethasone plasma cortisol levels is statistically significant $(r=0.45, p=0.000)$. The number of subjects in each category is in parentheses. 


\section{Discussion}

These data indicate that postdexamethasone plasma cortisol concentrations moderately reflect the degree of endogeneity, regardless of whether endogeneity is considered it continuous or a dichotomous variable. However, this correlation is not specific for "endogeneity" per se; DST values appear to reflect total severity of depression (HRSD total) even more strongly than endogeneity.

This relationship between severity of depression and the DST has received only sporadic attention, and most reports dealing with this question have considered DST results categorically, i.e., suppressors versus nonsuppressors. Carroll et al. (1968) initially reported an association between severity of depression and resistance to dexamethasone suppression, but in later reports Carroll and colleagues failed to conlim this relationship (Carroll and Davis 1970. Carroll et al. 1981). Brown et al. (1979) and Stokes et al. (1975) found that DST nonsuppressors were more severely depressed than suppressors. Davis et al. (1981) also noted that HRSD severity scores correlated positively and significantly with postdexamethasone cortisol concentrations; in contrast, predexamethasone baseline cortisol levels did not correlate with severity of depression. Brown and Shuey (1980) suggested that nonsuppressors have greater cognitive impairment than suppressors, based on their inability to complete self-rating forms, but these authors felt that suppressors and nonsuppressors did not differ significantly in severity of depression as measured by HRSD scores. Brown and Qualls (1981) also contended that nonsuppressors and suppressors did not differ in either severity or symptomatology. Thus, there are conflicting viewpoints concerning this issue when DST values are considered only categorically.

Few studies have analyzed DST cortisol levels on a continuum, such as described in this report. Reus (1982) showed that patients with high levels of predexamethasone 8:(N) $\mathrm{AM}$ cortisol were more symptomatic on admission than those with normal levels, partic ularly in areas of anxiety and somatic concerns. Starkman and Schteingart (1981) studied patients with Cushing's syndrome and observed a statistically significant relationship between the overall neuropsychiatric disability score and the degree of cortisol elevation. whether measured by cortisol secretion rate, urinary free cortisol, or plasma cortisol, at 8:00 AM. They also suggested that the relative proportion of cortisol to adrencorticotrophin hormone $(\mathrm{ACTH})$, rather than the level of either hormone itself, seemed to be related most closely to severity of "depressed mood." Feinberg and Carroll (1984) agreed that the 4:00 PM sample of plasma cortisol correlated significantly with HRSD scores. Thus. there is a growing concensus that absolute DST values do reflect severity of depression.

Recent weight loss has been suggested to alter hypothalamic-pituitary-adrenal function (Berger et al. 1982; Edelstein et al. 1983). Feinberg and Carroll (1984) demonstrated that weight loss is highly confounded with total severity and age. This is predictable, of course, because weight and appetite changes are integral components of major depression. Our data suggest that absolute weight may be as important a predictor of DST as reported weight loss per se, perhaps because the latter is so unreliable.

In addition to confirming previously reported relationships between DST values and endogeneity, these data dramatize several aspects of endogeneity. First, it may be essential to consider endogeneity on a continuum, as well as categorically. Although clinicians are inclined to consider many clinical concepts as "present" or "not present," "endo. geneity" more accurately may reflect a continuous degree of limbic pathophysiology. manifesting itself in increasing disturbances of reward reinforcement (anhedonia; loss of reactivity), sleep (initial. middle, or late insomnia; hypersomnia), psychomotor agitation 
or retardation; loss of, decreases in, or increases in libido, appetite (and thus weight changes), and chronobiological dysrythmias (diurnal mood variation). The DST, which is also regulated by the same limbic-hypothalamic regulatory regions, appears to parallel this severity of endogenous/limbic dysregulation.

Our data also confirm that endogeneity is confounded with total severity of depression. Even clinical items not traditionally considered to be "endogenous" are correlated with endogeneity. Caution is required in interpreting some of our data, given the small population of the nonendogenous group. These data suggest that considerable information is lost when clinical profiles are only considered dichotomously.

We thank Pamela Flegel, Senior Research Associate, and the nursing staff on the Clinical Studies Unit for their assistance in conducting this study.

\section{References}

Berger M, Doerr P, Lund R, Bronisch T, Von Zerssen D (1982): Neuroendocrinological and neurophysiological studies in major depressive disorders: Are there biological markers for the endogenous subtype? Biol Psychiatry 17:1217-1242.

Brown WA, Shuey I (1980): Response to dexamethasone and subtype of depression. Arch Gen Psychiatry 37:747-751.

Brown WA, Qualls CB (1981): Pituitary-adrenal disinhibition: Marker of a subtype with characteristic clinical features and response to treatment? Psychiatry Res 4:115-128.

Brown WA, Johnston R, Mayfield D (1979): The 24-hour dexamethasone suppression test in a clinical setting: relationship to diagnosis, symptoms, and response to treatment. Am J Psychiatry 136:543-547.

Carroll BJ (1982): The dexamethasone suppression test for mclancholia. Br J Psychiatry 140:292-304.

Carroll B, Davis BM (1970): Clinical association of 11-hydroxycorticosteroid suppression and nonsuppression in severe depression. Br Med J 1:789-791.

Carroll B, Martin FIR, Davies B (1968): Resistance to supression by dexamethasone of plasma 11-OHCS levels in severe depressive illness. Br Med J 3:285-287.

Carroll BJ, Curtis GC, Mendels J (1976): Neuroendocrine regulation in depression, II. Discrimination of depressed from non-depressed patients. Arch Gen Psychiatry 33:1051.

Carroll BJ, Greden JF, Feinberg M (1980): Neuroendocrine dysfunction in genetic subtypes of primary unipolar depression. Psychiatry Res 2:251-258.

Carroll BJ, Feinberg M, Greden JF, Tarika JS, Albala AA, Haskett RF, James NMcI, Krofol Z, Lohr N, Steiner M, DeVigne JP, Young E (1981): A specific laboratory test for the diagnosis of melancholia: Standardization, validation and clinical utility. Arch Gen Psychiatry 38:15-22.

Coryell W, Gaffney G, Burkhardt PE (1982): DSM-III. Melancholia and the primary-secondary distribution: A comparison of concurrent validity by means of the dexamethasone suppression test. Am J Psychiatry 139:120-122.

Davis KL, Hollister LE, Mathe AA, Davis BM, Rothpearl AB, Baull KF, Hsieh JVK, Barchas JD, Berger PA (1981): Neuroendocrine and neurochemical measurements in depression. Am J Psychiatry 138:1555-1562.

Edelstein CK, Roy-Byme P, Fawzy FI, Dornfeld L (1983): Effects of weight loss on the dexamethasone suppression test. Am J Psychiatry 140:338-341.

Feinberg M, Carroll B (1984): Biological markers for endogenous depression. Arch Gen Psychiarry 41:1080-1085.

Hamilton MA (1960): A rating scale for depression. I Neurol Neurosurg Psychiatry 23:56-62. 
Lewis DA, Winokur G (1983): 'The familial classification of primary unipolar depression: Biologica! validation of distinct subtypes. Comp Psychiatry 26:495-501

Murphy BEP (1967): Some studies of the protein binding of steroids and their application to the routine micro and ultra micro measurements of various steroids in body fluids by competitive protein-binding ratio assay. J Clin Endocrinol Metab 27:973-990.

Nasr SJ, Pandey G, Altman EG, Gibbons R, Gauiria FM, Davis J (1983): Symptom profile of patients with positive DST: A pilot study. Biol Psychiatry 18:571--574

Reus VI (1982): Pituitary-adrenal disinhibition as the independent variable in the assessment of behavioral symptoms. Biol Psychiatry 17:317-326

Rudorfer MV, Hai-Gwo Hwu. Clayton P (1982): Dexamethasone Suppression Test in primary depression: Significance of family history and psychosis. Biol Psvchiatry 17:41 48 .

Schlesser MA. Winokur G, Sherman BM (1979): Genetic subtypes of unipolar primary depression distinguished by hypothalamic-pituitary-adrenal ax is activity. Lancet $1: 739$

Spitzer RL, Endicott J (1975): The Schedule for Affective Disorders and Schizophrenia. New York: Biometrics Research Division. New York State Psychiatric Institute.

Spitzer RL. Endicott J. Robins E (1977): Research Diagnostic Criteria for a Selected Group of Functional Disorders, ed 3. New York: Biometrics Research Division. New York State Psy. thiatric Institute.

Starkman M, Schteingart H (1981): Neuropsychiatric manifestations of patients with Cushing "S syndrome. Arch Intern Med 141:215.

Starkman M. Schteingart H. Schork A (1981): Depressed mood and other psychiatric manifestations of Cushing's syndrome: Relationship to hormone levels. Psychosom Med 43:3-17

Stokes P. Pick (IR, Stoll PM (1975): Pituitary-adrenal function in depressed patients: Resistance to dexamethasone suppression. J Psychiat Res 12:271-281.

Winokur G, Behar D, Van Valkenberg C, Lowry M (1978): Is a familial definition of depression both feasible and valid. J Nerv Ment Dis 166:764-768. 